\title{
Genetically altering the expression of neutral trehalase gene affects conidiospore thermotolerance of the entomopathogenic fungus Metarhizium acridum
}

Yajun Leng ${ }^{1 \dagger}$, Guoxiong Peng ${ }^{1,2,3 \dagger}$, Yueqing Cao ${ }^{1,2,3}$, Yuxian Xia ${ }^{1,2,3^{*}}$

\begin{abstract}
Background: The entomopathogenic fungus Metarhizium acridum has been used as an important biocontrol agent instead of insecticides for controlling crop pests throughout the world. However, its virulence varies with environmental factors, especially temperature. Neutral trehalase (Nt) hydrolyzes trehalose, which plays a role in environmental stress response in many organisms, including M. acridum. Demonstration of a relationship between $\mathrm{Ntl}$ and thermotolerance or virulence may offer a new strategy for enhancing conidiospore thermotolerance of entomopathogenic fungi through genetic engineering.

Results: We selected four Ntl over-expression and four Nt/ RNA interference (RNAi) transformations in which $\mathrm{Ntl}$ expression is different. Compared to the wild-type, NtI mRNA expression was reduced to $35-66 \%$ in the RNAi mutants and increased by 2.5-3.5-fold in the over-expression mutants. The RNAi conidiospores exhibited less trehalase activity, accumulated more trehalose, and were much more tolerant of heat stress than the wild-type. The opposite effects were found in conidiospores of over-expression mutants compared to RNAi mutants. Furthermore, virulence was not altered in the two types of mutants compared to the wild type.
\end{abstract}

Conclusions: $\mathrm{Nt/}$ controlled trehalose accumulation in M. acridum by degrading trehalose, and thus affected conidiospore thermotolerance. These results offer a new strategy for enhancing conidiospore thermotolerance of entomopathogenic fungi without affecting virulence.

\section{Background}

Metarhizium acridum is a haploid entomopathogenic fungus (Hypocreales: Clavicipitaceae). M. acridum isolates have been used as biocontrol agents for crop pests, including sugar cane grubs, termites, cockroaches, and rhinoceros beetles [1]. M. acridum was commercialized and used for locust control in Australia, West Africa [2], and China [3].

Insecticide resistance, pest resurgence, and concerns over environmental impact have made the search for alternative means of biological pest control more urgent. Unfortunately, large-scale use of fungal biocontrol

\footnotetext{
* Correspondence: yuxianxia@cqu.edu.cn

† Contributed equally

'Genetic Engineering Research Center, College of Bioengineering,

Chongqing University, Chongqing, 400030, China

Full list of author information is available at the end of the article
}

agents is partially limited by the failure of conidia to retain virulence during long-term storage, transportation, and use under stressful conditions, such as high temperature, low humidity, and sunlight exposure [4-6]. Manipulation of culture conditions could optimize the concentration of spore polyols and sugars, including trehalose, and consequently increase tolerance to low relative humidity $[7,8]$. However, genetic manipulations of these polyols and sugars to enhance environmental tolerance have not been explored in entomopathogenic fungi.

To genetically engineer more robust entomopathogenic fungi, we focused on the trehalose pathways involved in stress response. Trehalose is a storage carbohydrate as trehalose concentrations are high when nutrients are limited in resting cells. In many microorganisms and invertebrate animals, trehalose plays a role

\section{C) Biomed Central}


in environmental stress response $[9,10]$ and is a known stress metabolite as its concentration increases during certain adverse environmental conditions, such as exposure to heat or toxic chemicals [11]. In Saccharomyces cerevisiae, trehalose is required for cells to survive diverse stresses, such as heat shock, starvation, and desiccation [12]. Additionally, it has been shown to provide one way for cells to survive thermal stress in vitro [13]. Based on the stress-protection properties of trehalose in vitro and the positive correlation between trehalose concentration and stress resistance in vivo, it is reasonable to expect that trehalose might function as a protective agent against stress $[14,15]$.

However, studies investigating the relationship between trehalose and thermotolerance have shown conflicting results. In S. cerevisiae, the trehalose level was positively correlated with stress resistance in different strains, growth conditions, and heat treatments [16-18]. Almost all strains exhibited more than a 2- to 10-fold increase in trehalose level after heat-shock treatment $[19,20]$. Additionally, the defective mutant of the neutral trehalase gene $(\mathrm{Ntl})$ produced organisms that were more thermotolerant than the wild type, most likely because of higher trehalose levels [21]. In contrast, some studies found no correlation between trehalose accumulation and thermotolerance under certain conditions, suggesting that trehalose may not mediate thermotolerance [22,23].

In most fungal species, trehalose hydrolysis is carried out by trehalase [24]. The single known exception is Pichia fermentans, in which trehalase has phosphorylase activity [25]. Fungal trehalases are classified into two categories according to their optimum $\mathrm{pH}$ : acid trehalases or neutral trehalases [26,27]. Cytosolic neutral trehalase degrades intracellular trehalose. The Ntl of S. cerevisiae, Kluyveromyces lactis, Candida utilis, Torulaspora delbrueckii, Schizosaccharomyces pombe, and Pachysolen tannophilus is tightly controlled by signaling pathways that end with the trehalose being reversibly activated by phosphorylation [27]. These signaling pathways can be triggered in vivo by glucose, nitrogen sources, heat shock, and chemicals like protonophores, which produce intracellular acidulation. This enzyme has been thoroughly studied in filamentous fungi, such as Aspergillus nidulans, Neurospora crassa, and Magnaporthe grisea [21,28], but little is known about $M$. acridum neutral trehalase $(\mathrm{Ntl})$ beyond the sequence in two strains, $M$. roberstii ARSEF2575 [29,30] and CQMa102 [31]. Using these sequences and genetic manipulation tools, we can now determine how $\mathrm{Ntl}$ affects stress response in terms of thermotolerance and virulence.

Different fungal growth phases (budding, conidiation, and germination) are associated with trehalose accumulation or mobilization. Depletion of trehalose storage marks early germination of fungal spores [26]. In
Cryptococcus neoformans and other pathogenic fungi, the trehalose pathway is a selective fungicidal target for antifungal development $[28,32]$. It is not known whether $\mathrm{Ntl}$ is a virulence factor in M. acridum.

We report here the construction of RNA interference (RNAi) and over-expression mutants of $\mathrm{Ntl}$ to investigate its role in thermotolerance and virulence of $M$. acridum. The results offer a new strategy for improving the thermotolerance of fungal conidia and yield insights into M. acridum spore physiology.

\section{Results}

\section{Over-expression and RNA interference mutants and the} expression of $\mathrm{Nt}$

The pBarEx-NTL over-expression vector contained a 2,535-nucleotide sequence from the $N t l$ genomic DNA fragment, including the full coding sequence and parts of the promoter and terminator sequences (Figure 1A). The pDPB-NTL vector contained 435 nucleotides of the $\mathrm{Ntl}$ coding sequence (Figure $1 \mathrm{~B}$ ). Both constructs were transformed to M. acridum CQMa102 using microparticle bombardment. Four M. acridum transformants for each construct were selected according to their ability to grow on selective media. PCR analysis showed that the vector was integrated into the fungal genome.

Expression of $\mathrm{Ntl}$ was analyzed by real-time PCR (Figure 2). In over-expression transformants, $N t l$ levels were 2.5-3.5-fold higher than in wild-type levels. In contrast, $\mathrm{Ntl}$ expression in RNAi transformants was reduced to $35-66 \%$ of wild-type levels.

\section{$\mathrm{Nt}$ is related to trehalose accumulation in conidia}

The neutral trehalase activity of conidia increased significantly in over-expression mutants compared to the wild-type strain and was reduced significantly in RNAi mutants $(\mathrm{p}<0.05)$ (Table 1$)$. Significantly positive correlation (correlation coefficient $=-0.816, \mathrm{p}<0.05$ ) was established between neutral trehalase activity and $N t l$ expression levels (Table 2). In contrast, the trehalose concentration in the wild-type strain was significantly higher than that in the over-expression mutants and

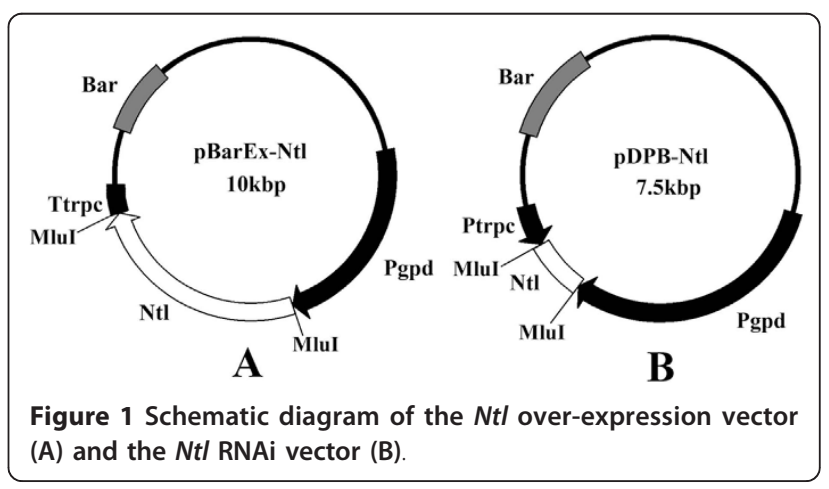




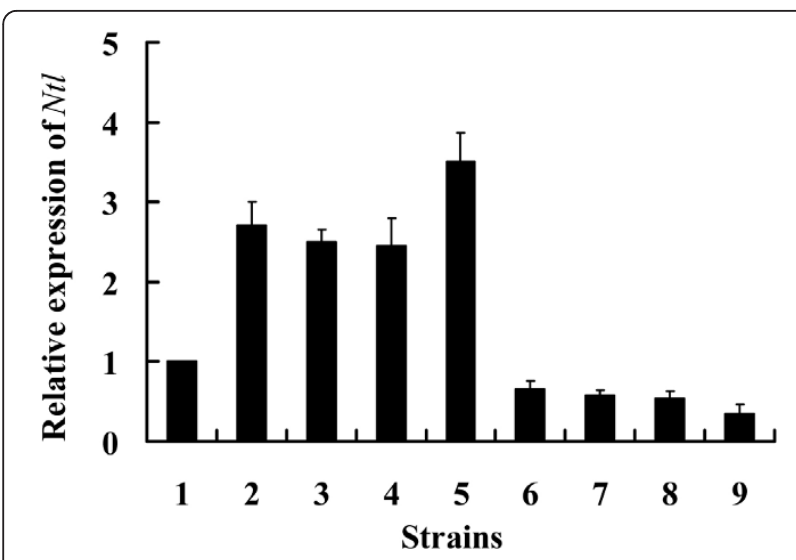

Figure 2 Real-time PCR analysis for relative expression of $\mathrm{NtI}$. 1: wild-type strain; 2-5: over-expression mutants; 6-9: RNAi mutants. Gapdh was analyzed in parallel as a loading control (not shown). Standard error (SE) bars are averages for three independent experiments.

lower than that in the RNAi mutants $(\mathrm{P}<0.05)$. This showed that the neutral trehalase activity varied inversely with the trehalose concentration in conidia. Furthermore, the trehalose concentration was significantly positively correlated with $\mathrm{Ntl}$ expression levels and neutral trehalase activity $(\mathrm{p}<0.05)$ (Table 2). This demonstrated that $N t l$ is related to trehalose accumulation because it controls the neutral trehalase activity.

\section{$\mathrm{Ntl}$ affects conidiospore thermotolerance}

After wet-heat exposure at $45^{\circ} \mathrm{C}$, the germination rate of conidia declined with increasing exposure time and the conidia germination rates of the wild-type strain and mutants appeared to be significantly reduced for each succeeding 0.5-hour interval (Figure 3). However, the response to tolerance was obviously different for the

Table 1 Trehalose concentrations and neutral trehalase activity in wild-type strain compared to over-expression mutants and RNAi mutants

\begin{tabular}{lllll}
\hline Strains & \multicolumn{2}{c}{$\begin{array}{c}\text { Trehalose (pg/ } \\
\text { conidium)* }\end{array}$} & \multicolumn{2}{c}{$\begin{array}{c}\text { Neutral trehalase activity (U/mg } \\
\text { protein)* }\end{array}$} \\
\hline 1 & $7.17 \pm 0.93$ & $\mathrm{c}$ & $14.28 \pm 1.14$ & $\mathrm{c}$ \\
\hline 2 & $5.04 \pm 1.17$ & $\mathrm{e}$ & $18.08 \pm 1.15$ & $\mathrm{ab}$ \\
\hline 3 & $6.10 \pm 0.22$ & $\mathrm{~d}$ & $16.43 \pm 1.21$ & $\mathrm{~b}$ \\
\hline 4 & $5.91 \pm 0.27$ & $\mathrm{de}$ & $16.29 \pm 1.15$ & $\mathrm{~b}$ \\
\hline 5 & $5.51 \pm 0.53$ & $\mathrm{e}$ & $16.12 \pm 0.96$ & $\mathrm{~b}$ \\
\hline 6 & $9.72 \pm 0.14$ & $\mathrm{~b}$ & $8.82 \pm 1.26$ & $\mathrm{~d}$ \\
\hline 7 & $10.76 \pm 0.83$ & $\mathrm{a}$ & $7.59 \pm 0.99$ & $\mathrm{e}$ \\
\hline 8 & $10.38 \pm 0.83$ & $\mathrm{ab}$ & $8.33 \pm 1.12$ & $\mathrm{de}$ \\
\hline 9 & $10.57 \pm 1.31$ & $\mathrm{ab}$ & $8.23 \pm 1.39$ & $\mathrm{de}$ \\
\hline
\end{tabular}

* Means $( \pm \mathrm{SE})$ of 3 repetitions followed by different lowercase letters in the same column were significantly different at the $p<0.05$ level according to the ANOVA table and Tukey's multiple range test. 1: wild-type strain; 2-5: over-expression mutants; 6-9: RNAi mutants.
Table 2 Correlation coefficients (R) of treatments and cellular components

\begin{tabular}{lllll}
\hline & Dry-heat $(R)$ & Wet-heat(R) & Trehalose(R) & mRNA(R) \\
\hline mRNA & -0.9818 & -0.890 & -0.831 & 1.000 \\
Trehalose & 0.873 & 0.898 & 1.000 & -0.831 \\
Trehalase & -0.889 & -0.905 & -0.867 & 0.816 \\
\hline
\end{tabular}

wild-type strain, over-expression mutants, and RNAi mutants. The conidia germination rate of the wild-type strain was significantly higher than that of the overexpression mutants $(\mathrm{p}<0.05)$ and lower than that of the RNAi mutants $(\mathrm{p}<0.05)$. Similar results were observed after dry-heat exposure at $65^{\circ} \mathrm{C}$ for $0,1,2,3$, 4 , or 5 hours. Accordingly, the inhibition time value for $50 \%$ germination $\left(\mathrm{IT}_{50}\right)$ of the wild-type strain was longer than that of the over-expression mutants $(\mathrm{p}<$ $0.05)$ and shorter than that of the RNAi mutants ( $\mathrm{p}<$ 0.05 ) (Figure 4). These data showed that the $N t l$ overexpression mutants were significantly more sensitive to heat compared with the wild-type strain $(\mathrm{p}<0.05)$. Contrary to that of the over-expression mutants, the thermotolerance of the Ntl RNAi mutants was significantly higher than that of the wild-type strain $(\mathrm{p}<0.05)$.

Furthermore, both trehalase and Ntl mRNA levels were negatively correlated with the germination rates of conidia treated with wet heat and dry heat $(\mathrm{p}<0.05)$ (Table 2), suggesting that $N t l$ affects conidiospore thermotolerance.

\section{$N t /$ has no effect on virulence}

Bioassays revealed that mortality trends of locusts inoculated with over-expression mutants or RNAi mutants were similar to that of locusts inoculated with wild strain (Figure 5A). Accordingly, no significant differences were observed in locust lethal time values for $50 \%$ mortality $\left(\mathrm{LT}_{50}\right)$ between the wild-type strain, over-expression mutants, or RNAi mutants ( $p>0.05$ ) (Figure 5B). This result suggested changes in $N t l$ expression level did not affect the virulence of $M$. acridum.

\section{Discussion}

Resisting thermal stress is important for pathogens of the locust, like $M$. acridum, because temperatures fluctuate in locust habitats and locusts themselves could also employ behavioral fever to counter fungal infection [33]. $N t l$ has been reported to play an important role in environmental stress response. In this study, the function of $\mathrm{Ntl}$ with respect to thermotolerance in $\mathrm{M}$. acri$d u m$ was investigated by changing its expression level via RNAi and over-expression mutants.

Trehalose is an important factor determining thermotolerance in $M$. acridum. Trehalose content and thermotolerance were significantly and positively correlated, and $\mathrm{Ntl}$ activity was significantly and negatively correlated with 

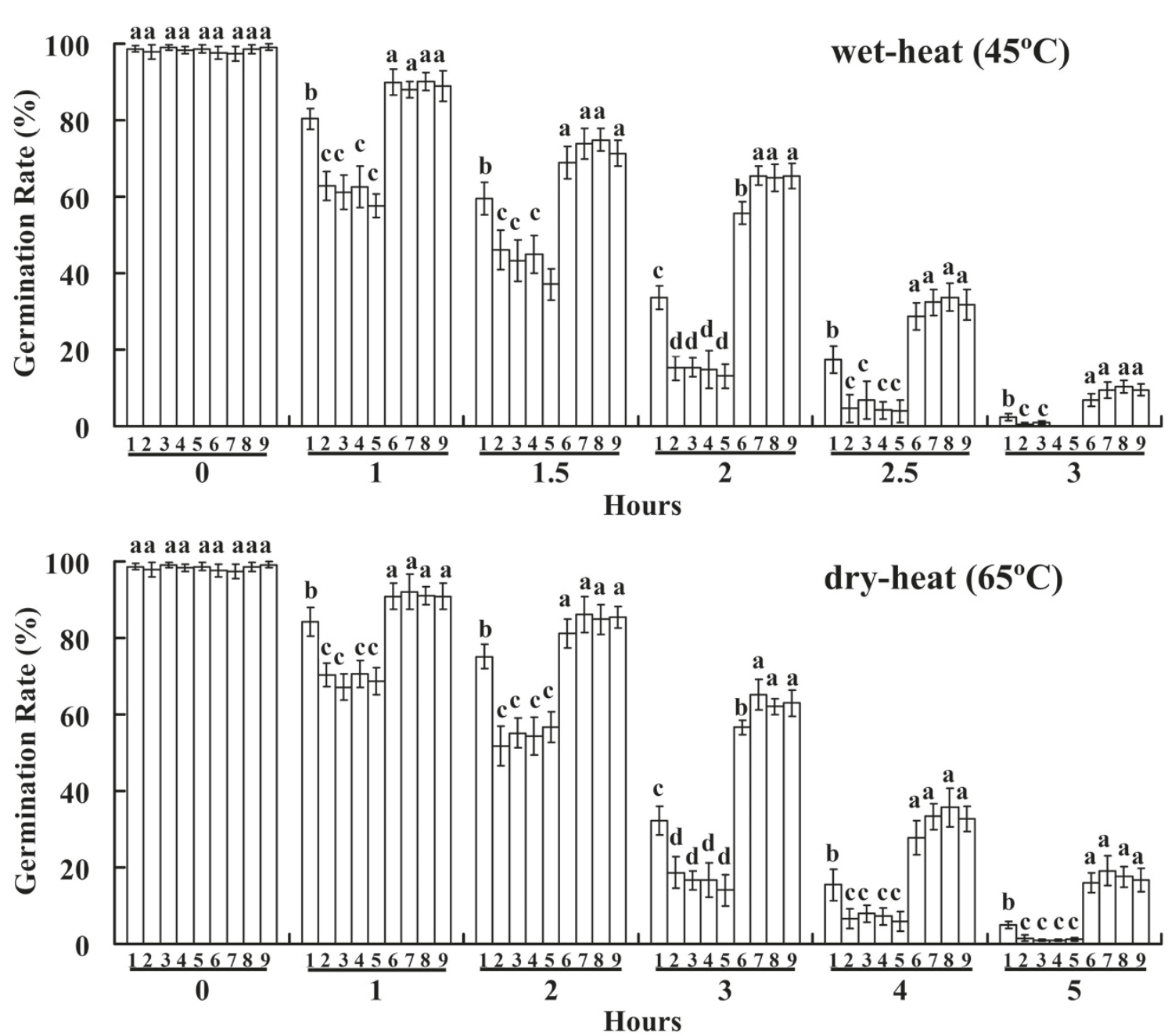

Figure 3 Germination rates of $\mathbf{M}$. acridum wild-type strain and $\mathbf{N t /}$ mutants. Wet-heat: aqueous conidial suspensions exposed to $45^{\circ} \mathrm{C}$ for 0 , $0.5,1,1.5,2$, or 2.5 hours; dry-heat: dried conidia exposed to $65^{\circ} \mathrm{C}$ for $0,1,2,3,4$, or 5 hours. 1: wild-type strain; 2-5: over-expression mutants; 6-9: RNAi mutants. Standard error bars (SE) show averages for three independent experiments. Significant differences are designated by the lowercase letters on the bars of each group $(p<0.05)$.

thermotolerance (Table 2). These results suggest that trehalose accumulation and metabolism play important roles in thermotolerance, but this factor is not the only controller of thermotolerance [22,34]. The accumulation and metabolism of other polyols, such as sucrose and glycerol, may also be factors in stress response [22]. It is possible that changes in trehalose concentration produced by upor down-regulating trehalase levels may also affect the levels of other polyols and the entire metabolic process. Further investigation of other polyols in the Ntl mutants is required to understand fully the mechanism of the effect of $\mathrm{Ntl}$ on $\mathrm{M}$. acridum thermotolerance.

Field conditions and abiotic environmental factors, such as temperature, moisture, and sunlight, influence whether infection can occur. When the host temperature favors a short germination time and that temperature is above or below the pathogen's optimum, temperature can be a limiting factor for the disease. However, oil-based formulations and selective media have been shown to enhance the thermotolerance of $M$. acridum conidia, resulting in promising acridid control in the field $[35,36]$. Using the genetic manipulation tools introduced here for $M$. acridum, the thermotolerance of the mycoinsecticidal strain will be improved to allow for wider commercial application.

A secretary trehalase activity of $M$. acridum was detected in the hemolymph of infected insects, suggesting that it is a virulence factor in insect pathogenesis [29]. In contrast, the changes in neutral trehalase expression had no effects on virulence in this study, which agrees with the report on C. neoformans that a neutral trehalase mutant does not possess any known virulence defects [32]. Our results indicate that trehalose 


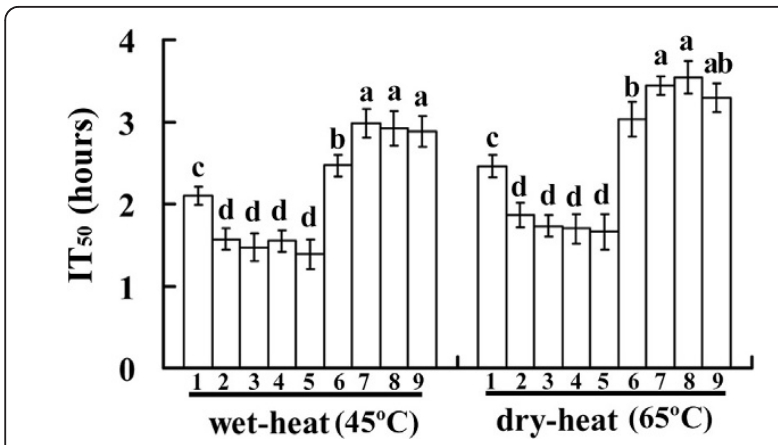

Figure $4 \mathrm{IT}_{50}$ of $M$. acridum wild-type strain and $\mathrm{Nt}$ mutants. $\mathrm{IT}_{50}$ : inhibition time values for $50 \%$ germination of aqueous conidial suspensions exposed to $45^{\circ} \mathrm{C}$ and dried conidia exposed to $65^{\circ} \mathrm{C}$, respectively. 1: wild-type strain; 2-5: over-expression mutants; 6-9: RNAi mutants. Standard error (SE) bars show averages for three independent experiments. Significant differences are designated by the different lowercase letters on the bars of each group in the wet-heat or dried-heat test $(p<0.05)$.
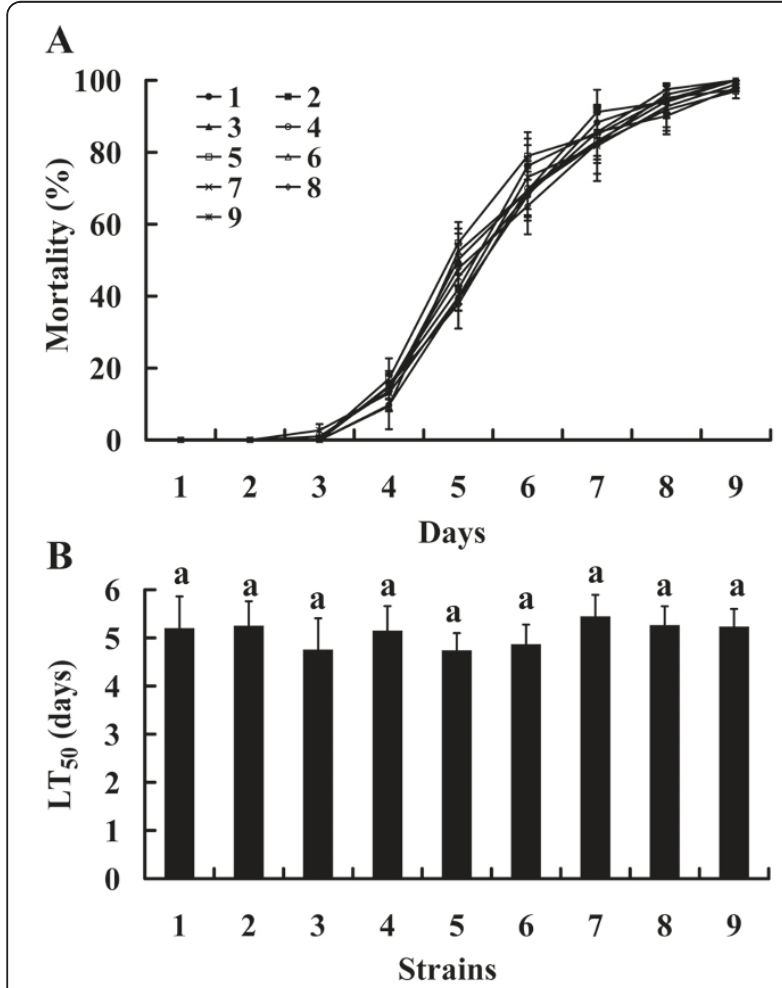

Figure 5 Bioassay results for $M$. acridum against Locusta migratoria. 1: wild-type strain; 2-5: over-expression mutants; 6-9: RNAi mutants. A: mortality $( \pm \mathrm{SE})$ of Locusta migratoria treated with wild-type strain and various $\mathrm{Nt} /$ transformants; B: lethal time values for $50 \%$ mortality $\left(L T_{50}\right)$ values of Locusta migratoria treated with wild-type strain and various Nt/ transformants. Standard error (SE) bars are averages for four independent experiments. Same lowercase letters indicate no significant differences ( $p>0.05)$. in conidia does not affect virulence; thus, genetically engineering the trehalose pathway would increase the thermotolerance of fungal strains with no loss of virulence. Temperature tolerance also affects fungal agent storage longevity [4]. Further studies are required to investigate the longevity of the mutants.

The dual promoter RNAi system developed in this study successfully knocked down the gene expression in filamentous fungus. In previous studies, genes that were knocked down with isopliae over-expression and RNAi Ntl transformants exhibited no loss in virulence compared to wild-type silencing vectors that produced hairpin or intron-containing hairpin RNA in fungi [37-43], which involved two steps of oriented cloning. The dual promoter system simplified the RNAi construction procedure to one single-step non-oriented cloning, in which transcription of a target gene from each promoter produced a pool of sense and antisense RNAs in the cells. This system provides an easy and efficient tool for knocking down gene expression, and can be extended to knock down multiple gene targets from transcriptionally fused genes. Thus, the dual promoter system offers an efficient platform for functional analysis of entomopathogenic fungal genes and genetic manipulation for strain improvement.

\section{Conclusions}

Our study shows that $N t l$ expression of $M$. acridum can be effectively enhanced or inhibited by over-expression or RNAi mutants, respectively, using a dual promoter system. Compared to the wild-type, $N t l$ mRNA was reduced to $35-66 \%$ in RNAi mutants and increased by 2-3-fold in the over-expression mutants. The conidiospores of RNAi mutants had less trehalase activity, accumulated more trehalose, and were much more tolerant of heat stress than the wild type. The opposite effects were found in conidiospores of over-expression mutants compared to RNAi mutants. The $N t l$ mRNA level was positively correlated with neutral trehalase activity and negatively correlated with trehalose concentration and the thermotolerance of conidiospores, further confirming the role of $\mathrm{Ntl}$ in the thermotolerance of $\mathrm{M}$. acridum. Furthermore, bioassays showed that alteration of $\mathrm{N} t \mathrm{l}$ expression did not affect the virulence.

In conclusion, $\mathrm{N} t \mathrm{l}$ regulates thermotolerance through trehalose accumulation in $M$. acridum but does not affect its virulence. The use of the RNAi mutant of $N t l$ could provide a new strategy for improving the conidiospore thermotolerance of an entomopathogenic fungus without compromising its virulence.

\section{Methods}

\section{Strain growth conditions}

M. acridum strain CQMa102, a locust-specific strain, was isolated by our laboratory in Chongqing, China. 
Conidia were harvested from cultures grown on $1 / 4$ strength Sabouraud's dextrose agar medium (SDA: $1 \%$ dextrose, $0.25 \%$ mycological peptone, $2 \%$ agar, and $0.5 \%$ yeast extract) at $28^{\circ} \mathrm{C}$. Mycelia for DNA and RNA extraction were grown by inoculating $100 \mathrm{~mL} \mathrm{1/4.} \mathrm{SDA}$ liquid media with $10^{6}$ conidia and incubating at $28^{\circ} \mathrm{C}$ with shaking at $150 \mathrm{rpm}$ for 2-3 days.

\section{Construction of the $\mathrm{Nt}$ l over-expression vector}

An over-expression vector ( $\mathrm{pBarEx}$ ) for filamentous fungi was constructed based on pBTM. pBarEx contained a bar gene, promoter pGpdA, and terminator $\mathrm{TTrpC}$ from $A$. nidulans and a polylinker between pGpdA and TTrpC.

The full cDNA sequence of $N t l$ was amplified using Pyrobest DNA polymerase (TaKaRa, Japan) with primers B1 (5'-AAT TAC GCG TAC CTC CAC GTT CGT CAG TC-3' with an MluI recognition sequence at the 5' end) and B2 (5'-CGC CAC GCG TTT GAG AGG GCA ATT AAT CG-3' with an MluI recognition sequence at the 3' end). The PCR product and vector pBarEx were both digested with $M l u I$, and then ligated using T4 DNA ligase (pBarEx-NTL, Figure 1A).

\section{Construction of the NtI RNAi vector}

A dual promoter RNAi vector for filamentous fungi was first constructed based on pBTM, which was reported previously [44], pDPB containing a selectable marker, the bar gene (resistance to ammonium glufosinate), polylinker, and two promotors in opposite direction (pGpdA and pTrpC from A. nidulans).

A fragment of the coding sequence of Ntl (310-745) was then amplified from $M$. acridum Ntl cDNA with primers A1 (5'-ATT AAC GCG TAG CAC AAG AAG ATA CCG ATG-3' with an MluI restriction site at the 5' end) and A2 (5'-TAT AAC GCG TCG CGC CAG GGA GCT GCT GGA CAT CTAG-3' with an MluI restriction site at the 3 ' end), which was designed according to the CQMa102 Ntl cDNA sequence (GenBank AY557612). The PCR product and vector pDPB were both digested with MluI, and then ligated using T4 DNA ligase (Takara, Japan) (pDPB-NTL) (Figure 1B).

\section{Transformation of $M$. acridum}

Intact $M$. acridum CQMa102 conidia were transformed by microparticle bombardment (Model PDS-1000/He biolistic particle delivery system, Bio-Rad, USA). For bombardment, $50 \mu \mathrm{L}$ of conidia suspension $\left(10^{9}\right.$ coni$\mathrm{dia} / \mathrm{mL}$ ) were placed in the center of a Petri dish. Plasmids pDPB-NTL and pBarEx-NTL were linearized with BamHI and bound to $0.6-\mu \mathrm{m}$ diameter golden particles and then transformed into $M$. anisoplia by particlemediated DNA delivery (Model PDS-1000/He biolistic particle delivery system, Bio-Rad, USA), according to St
Leger [45]. Following bombardment, conidia were resuspended in $5 \mathrm{~mL}$ of MilliQ water. Aliquots of $200 \mu \mathrm{L}$ were plated on Czapek's medium (3\% saccharose, $0.2 \%$ $\mathrm{NaNO}_{3}, 0.1 \% \mathrm{~K}_{2} \mathrm{HPO}_{4}, 0.05 \% \mathrm{KCl}, 0.05 \% \quad \mathrm{MgSO}_{4}$, $0.001 \% \mathrm{FeSO}_{4}$ ) containing $200 \mu \mathrm{g} / \mathrm{mL}$ ammonium glufosinate and incubated at $27^{\circ} \mathrm{C}$ for 6-8 days. Transformants were confirmed by PCR amplification of bar gene. Post-transformation mitotic stability was evaluated according to the method in a previous report [46].

\section{Quantification analysis of $\mathbf{N t}$ transcript}

Total RNA was isolated from mycelia using the Trizol reagent (Invitrogen, USA). The cDNA was synthesized from DNaseI-treated total RNA with an anchored oligo-dT primer following the manufacturer's protocol (Promega, USA). Real-time PCR was performed using the SYBRGreen PCR Master Mix kit (Bio-Rad) in a Light Cycler (Bio-Rad). A standard curve was made to optimize the amplification efficiency with the primer pairs L1 (5'-GCACAAGAAGATACCGATGGC-3') and L2 (5'-CGATCCACTGGGTTCTCATTTA-3'). Gdpdh encoding gly ceraldehyde-3-phosphate dehydrogenase was selected as an internal control, and the primers of 5'-AGATGGAGGAGTTGGTGTTG-3' and 5'-GACTGCCCGCATTGAGAAG-3' were used for it [47]. The cycling conditions were $95^{\circ} \mathrm{C}$ for $3 \mathrm{~min}$ followed by 45 cycles of $95^{\circ} \mathrm{C}$ for 10 sec, annealing at $59^{\circ} \mathrm{C}(\mathrm{Ntl})$ or $60^{\circ} \mathrm{C}(\mathrm{Gdpdh})$ for $10 \mathrm{sec}$. The relative expression level of the $N t l$ in $M$. acridum transformants compared to that in wild-type strain was determined with the comparative cycle threshold $\left(C_{\mathrm{T}}\right)$ method [48]. Biological techniques were conducted in quadruplicate.

\section{Measurement of trehalose concentrations and trehalase activity}

Trehalose levels in conidia were measured using a method modified from Foster et al. [28]. Conidia of both wild-type and $M$. acridum transformants were harvested from 14 day plates, washed with distilled water, resuspended in $500 \mu \mathrm{L}$ of water, boiled for $20 \mathrm{~min}$, and disrupted by vortexing with glass beads $(0.5 \mathrm{~mm})$. Cell debris was removed by centrifugation at $13,000 \mathrm{~g}$ for $5 \mathrm{~min}$ and the supernatant was stored at $0^{\circ} \mathrm{C}$ prior to trehalose assay. A $50-\mu \mathrm{L}$ aliquot of the conidia lysis solution was added to $50 \mu \mathrm{L}$ of $0.1 \mathrm{M}$ sodium citrate buffer ( $\mathrm{pH}$ 5.6). Duplicate samples were incubated with or without $10 \mu \mathrm{L}$ porcine kidney acidic trehalase (Sigma, USA) overnight at $37^{\circ} \mathrm{C}$. The reaction was stopped by boiling the sample for $10 \mathrm{~min}$. Following centrifugation, the glucose concentration in the supernatant was assayed via a glucose assay kit (Bioscience, China).

To assay trehalase activity, $25 \mu \mathrm{L}$ of the trehalase extraction solution were added to the trehalose solution containing $50 \mathrm{mM}$ HEPS, and the mixture was incubated 
for $30 \mathrm{~min}$ at $37^{\circ} \mathrm{C}$. The reaction was stopped by boiling the samples for $10 \mathrm{~min}$, the samples were centrifuged, and the glucose in the supernatant was assayed using a commercial kit (Trinder, Sigma).

\section{Heat shock treatment}

Conidia were prepared as described above. For the wetheat shock test, conidia were suspended in $1 \mathrm{~mL}$ sterilized water. The suspension was vigorously shaken and filtered through cotton cloth and diluted to a concentration of $1 \times 10^{7}$ conidia. $\mathrm{mL}^{-1}$. Subsequently, the suspension was immediately placed in a stirred water bath at $45^{\circ} \mathrm{C}$ for $0.5,1,1.5,2$, or 2.5 hours. For the dry-heat shock test, conidia were dried in a desiccator containing silica gel until the moisture content was less than $5 \%$. Dried conidia were maintained in an incubator oven at $65^{\circ} \mathrm{C}$ for $1,2,3,4$, or 5 hours, and then suspended in sterilized water $\left(1 \times 10^{7}\right.$ conidia. $\left.\mathrm{mL}^{-1}\right)$. The conidial suspensions maintained at $28^{\circ} \mathrm{C}$ were used as a control. Germinations were measured by plating $50 \mu \mathrm{L}$ on $1 / 4$ SDA plates. After 24 hours incubation in the dark at $28^{\circ} \mathrm{C}$, the germination rate was checked with a microscope (Motic, china) at 400× magnification. About 300 conidia were evaluated for germination from different areas in each plate. Inhibition time values for $50 \%$ germination $\left(\mathrm{IT}_{50}\right)$ were used to estimate the conidiospore thermotolerance of M. acridum using DPS software [49].

\section{Bioassays}

Locusta migratoria were reared in our lab under crowded conditions as previously described by He et al. [50]. Male and female insects were separated after adult emergence. Male adult locusts (2-3 days after eclosion) were used in the bioassay tests. A $5-\mu \mathrm{L}$ solution of $2 \times$ $10^{6}$ conidia/mL of either wild-type $M$. acridum or transformants in cottonseed oil (Sigma) was applied to the locusts' head-thorax junctions. Treated locusts were separately confined in cages $(20 \times 20 \times 20 \mathrm{~cm})$ by 40 mesh, and kept at a temperature of $28^{\circ} \mathrm{C}$ with a $16: 8 \mathrm{~h}$ (light:day) photoperiod. There were four replications of $n$ $=30$ locusts in each treatment. Mortality was recorded daily and lethal time values for $50 \%$ mortality $\left(\mathrm{LT}_{50}\right)$ values were used to estimate the infectivity of M. acridum by DPS software [49].

\section{Statistical analysis}

All samples and treatments were carried out in triplicate unless stated otherwise. Data were square root arcsine transformed before being subjected to analysis of variance (ANOVA) for a completely randomized design. The means were separated using Tukey's multiple range test, carried out using DPS software [47]. Statistical significance was established at $\mathrm{p}<0.05$.

\section{Acknowledgements}

The research was supported by grants from the Natural Science Foundation of China (No. 30170630), and the Natural Science Foundation of Chongqing Sci-Tech Commission, P. R. China (No. 2008BB1178).

\section{Author details}

${ }^{1}$ Genetic Engineering Research Center, College of Bioengineering, Chongqing University, Chongqing, 400030, China. ${ }^{2}$ Chongqing Engineering Research Center for Fungal Insecticides, Chongqing, 400030, China. ${ }^{3}$ Key Lab of Functional Gene and Regulation Technologies under Chongqing Municipal Education Commission, Chongqing, 400030, China.

\section{Authors' contributions}

$Y X$ designed the study. $Y L, G P, Y C$, and $Y X$ wrote the manuscript. $Y L, G P, Y C$, and $Y X$ performed the experiments in this study. In particular, GP performed the data analysis and bioassay experiments, and YC participated in construction of the vector. All authors read and approved the final manuscript.

Received: 14 August 2010 Accepted: 10 February 2011 Published: 10 February 2011

\section{References}

1. Charnley AK, Collins SA: Entomopathogenic fungi and their role in pest control. Mycota: Environmental and Microbial Relationships 2007, 4:159-187.

2. Lomer C, Bateman R, Johnson D, Langewald J, Thomas M: Biological control of locusts and grasshoppers. Annu Rev Entomol 2001, 46:667-702.

3. Peng G, Wang Z, Yin Y, Zeng D, Xia Y: Field trials of Metarhizium anisopliae var. acridum (Ascomycota: Hypocreales) against oriental migratory locusts, Locusta migratoria manilensis (Meyen) in Northern China. Crop Prot 2008, 27:1244-1250.

4. Daoust RA, Roberts DW: Studies on the prolonged storage of Metarhizium anisopliae conidia: Effect of temperature and relative humidity on conidial viability and virulence against mosquitoes. J Invertebr Pathol 1982, 41:143-150.

5. Ekesi S, Maniania NK, Lux SA: Effect of soil temperature and moisture on survival and infectivity of Metarhizium anisopliae to four tephritid fruit fly puparia. J Invertebr Pathol 2003, 83:157-167.

6. Rangela DEN, Braga GUL, Flintc SD, Andersona AJ, Roberts DW: Variations in UV-B tolerance and germination speed of Metarhizium anisopliae conidia produced on insects and artificial substrates. J Invertebr Pathol 2004, 87:77-83.

7. Hallsworth JE, Magan N: Effect of carbohydrate type and concentration on polyhydroxy alcohol and trehalose content of conidia of three entomopathogenic fungi. Microbiology 1994, 140:2705-2713.

8. Hallsworth JE, Magan N: Manipulation of intracellular glycerol and erythritol enhances germination of conidia at low water availability. Microbiology 1995, 141:1109-1115.

9. Elbein A: The metabolism of alpha, alpha-trehalose. Adv Carbohydr Chem Biochem 1973, 30:227-256.

10. Thevelein JM: Regulation of trehalose metabolism and its relevance to cell growth and function. In The Mycota, Biochemistry and Molecular Biology. Volume 3. Edited by: Brambl R, Marzluf GA. Springe; 1996:395-420.

11. Nwaka S, Holze H: Molecular biology of trehalose and the trehalases in the yeast Saccharomyces cerevisiae. Prog Nucleic Acid Res Mol Biol 1998, 58:197-237.

12. Virgilio $C D$, Hottiger $T$, Dominguez J, Boller T, Wiemken $A$ : The role of trehalose synthesis for the acquisition of thermotolerance in yeast. I. Genetic evidence that trehalose is a thermoprotectant. Eur J Biochem 1994, 219:179-186

13. Hottiger $T$, Virgilio CD, Hall MN, Boller T, Wiemken A: The role of trehalose synthesis for the acquisition of thermotolerance in yeast 11 . Physiological concentrations of trehalose increase the thermal stability of proteins in vitro. Eur J Biochem 1994, 219:187-193.

14. Laere AV: Trehalose, reserve and/or stress metabolite? FEMS Microbio/ Rev 1988, 63:201-210.

15. Wiemken A: Trehalose in yeast, stress protectant rather than reserve carbohydrate. Antonie van Leeuwenhoek 1990, 58:209-217.

16. Attfield PV: Trehalose accumulates in Saccharomyces cerevisiae during exposure to agents that induce heat shock response. FEBS lett 1987, 225:259-263. 
17. Gélinas P, Fiset G, Leduy A, Goulet J: Effect of growth conditions and trehalose content on cryotolerance of bakers' yeast in frozen doughs. Appl Environ Microbiol 1989, 55:2453-2459.

18. Hottiger $T$, Boller $T$, Wiemken A: Rapid changes of heat and desiccation tolerance correlated with changes of trehalose content in Saccharomyces cerevisiae cells subjected to temperature shifts. FEBS lett 1987, 220:113-115.

19. Bonini BM, Neves MJ, Jorge JA, Terenzi HF: Effects of temperature shifts on the metabolism of trehalose in Neurospora crassa wild type and a trehalase-deficient (tre) mutant. Evidence against the participation of periplasmic trehalase in the catabolism of intracellular trehalose. Biochim Biophys Acta 1995, 1245:339-347.

20. Doehlemann G, Berndt P, Hahn M: Trehalose metabolism is important for heat stress tolerance and spore germination of Botrytis cinerea. Microbiology 2006, 152:2625-2634.

21. D'enfert C, Bonini BM, Zapella PDA, Fontaine T: Neutral trehalases catalyse intracellular trehalose breakdown in the filamentous fungi Aspergillus nidulans and Neurospora crassa. Mol microbiol 1999, 32:471-483.

22. Nwaka S, Kopp M, Burgert M, Deuchler I, Kienle I, Holzer H: Is thermotolerance of yeast dependent on trehalose accumulation? FEBS lett 1994, 344:225-228.

23. Nwaka S, Mechler B, Destruelle M, Holzer H: Phenotypic features of trehalase mutants in Saccharomyces cerevisiae. FEBS Lett 1995, 360:286-290.

24. Jorgea JA, Lourdes MD, Polizeli TM, Thevelein JM, Terenzi HF: Trehalases and trehalose hydrolysis in fungi. FEBS lett 1997, 154:165-71.

25. Schick I, Haltrich D, Kulbe KD: Trehalose phosphorylase from Pichia fermentans and its role in the metabolism of trehalose. Appl Microbiol Biotechnol 1995, 43:1088-1095.

26. Thevelein JM: Regulation of Trehalose mobilization in fungi. Microbio/ Rev 1984, 48:42-59.

27. Thevelein J: Regulation of trehalase activity by phosphorylation dephosphorylation during developmental transitions in fungi. Exp Mycol 1988, 12:1-12

28. Foster JA, Jenkinson JM, albot NJ: Trehalose synthesis and metabolism are required at different stages of plant infection by Magnaporthe grisea. EMBO J 2003, 22:225-235

29. Xia Y, Clarkson JM, Charnley AK: Trehalose-hydrolysing enzymes of Metarhizium anisopliae and their role in pathogenesis of the tobacco hornworm, Manduca sexta. J Invertebr Pathol 2002, 80:139-147.

30. Xia Y, Gao M, Clarkson J, Charnley AK: Molecular cloning, characterization, and expression of a neutral trehalase from the insect pathogenic fungus Metarhizium anisopliae. J Invertebr Pathol 2002, 80:127-137.

31. Hu Z, Wang Z, Peng G, Yin Y, Xia Y: Cloning and characterization of the neutral trehalase gene in Metarhizium anisopliae CQMa102. Acta Microbiologica Sinica 2005, 45:890-894.

32. Petzold EW, Himmelreich U, Mylonakis E, Rude T, Toffaletti D, Cox GM, Miller $J L$, Perfect JR: Characterization and regulation of the trehalose synthesis pathway and its importance in the virulence of Cryptococcus neoformans. Infect Immun 2006, 74:5877-5887.

33. Bundey S, Raymond S, Dean P, Roberts SK, Dillon RJ, Charnley AK: Eicosanoid involvement in the regulation of behavioral fever in the desert locust, Schistocerca gregaria. Arch Insect Biochem Physiol 2003, 52:183-192.

34. Nwaka S, Kopp M, Holzer H: Expression and function of the trehalase genes NTH1 and YBR0106 in Saccharomyces cerevisiae. J Bio Chem 1995 270:10193-10198.

35. Symmon P: Strategies to combat the desert locust. Crop Prot 1992, 11:25-28.

36. Bateman R: Methods of application of microbial pesticide formulations for the control of grasshoppers and locusts. Mem Entomol Soc Canada 1997, 171:69-81.

37. Liu H, Cottrell TR, Pierini LM, Goldman WE, Doering TM: RNA interference in the pathogenic fungus Cryptococcus neoformans. Genetics 2002, 160:463-470.

38. Kadotani N, Nakayashiki H, Tosa Y, Mayama S: RNA silencing in the phytopathogenic fungus Magnaporthe oryzae. Mol Plant Microbe Interact 2003, 16:769-775.

39. Fitzgerald A, Kan JA, Plummer KM: Simultaneous silencing of multiple genes in the apple scab fungus, Venturia inaequalis, by expression of RNA with chimeric inverted repeats. Fungal Genet Biol 2004, 41:963-971.
40. Mouyna I, Henry C, Doering TL, Latge JP: Gene silencing with RNA interference in the human pathogenic fungus Aspergillus fumigatus. FEMS Microbiol Lett 2004, 237:317-324.

41. Rappleye CA, Engle JT, Goldman WE: RNA interference in Histoplasma capsulatum demonstrates a roles for a-(1,3)-glucan in virulence. Mol Microbiol 2004, 53:153-165

42. McDonald T, Brown D, Keller NP, Hammond TM: RNA silencing of mycotoxin production in Aspergillus and Fusarium species. Mol Plant Microbe Interact 2005, 18:539-545.

43. Tanguay P, Bozza S, Breuil C: Assessing RNAi frequency and efficiency in Ophiostoma floccosum and O. piceae. Fungal Genet Biol 2006, 43:804-812.

44. Cao Y, Peng G, He Z, Wang Z, Yin Y, Xia Y: Transformation of Metarhizium anisopliae with benomyl resistance and green fluorescent protein genes provides a tag for genetically engineered strains. Biotechnol Lett 2007, 29:907-911.

45. St Leger RJ, Shimizu S, Joshi L, Biodochka MJ, Roberts DW: Co-transformation of Metarhizium anisopliae by electroporation or using the gene gun to produce stable GUS transformants. FEMS Microbiol Lett 1995, 131:289-29.

46. Goettel MS, Leger RJS, Bhairi S, Jung MK, Oakley BR, Roberts DW, Staples RC: Virulence and growth of Metarhizium anisopliae stably transformed to benomyl resistance. Curr Genet 1990, 17:129-132.

47. Peng GX, Xie L, Hu J, Xia YX: Identification of genes that are preferentially expressed in conidiogenous cell development of Metarhizium anisopliae by suppression subtractive hybridization. Curr Genet 2009, 55:263-271.

48. Livak KJ, Schmittgen TD: Analysis of relative gene expression data using real-time quantitative PCR and the 2(-delta Delta C(T)) method. Methods 2001, 25:402-408.

49. Tang QY, Feng MG: DPS Data Processing System for Practical Analysis Science Press, Beijing; 2002

50. He ZB, Cao YQ, Yin YP, Wang ZK, Chen B, Peng GX, Xia YX: Role of hunchback in segment patterning of Locusta migratoria manilensis revealed by parental RNAi. Dev Growth Differ 2006, 48:439-445.

doi:10.1186/1471-2180-11-32

Cite this article as: Leng et al:: Genetically altering the expression of neutral trehalase gene affects conidiospore thermotolerance of the entomopathogenic fungus Metarhizium acridum. BMC Microbiology 2011 11:32.

\section{Submit your next manuscript to BioMed Central and take full advantage of:}

- Convenient online submission

- Thorough peer review

- No space constraints or color figure charges

- Immediate publication on acceptance

- Inclusion in PubMed, CAS, Scopus and Google Scholar

- Research which is freely available for redistribution 